\title{
Endoscopic characterization of small colonic polyps: baseline performance of experienced endoscopists is no different to that of medical students
}

다(1)이우

\author{
Authors \\ Peter Basford', James Brown², Sarah Cooper², Pradeep Bhandari ${ }^{3}$ \\ Institutions \\ 1 Western Sussex Hospitals NSH Trust, St. Richard's \\ Hospital, Chichester PO19 5NY, UK \\ 2 Pharmacology and Biomedical Sciences, University of \\ Portsmouth, St. Michael's Building, White Swan Rd, \\ Portsmouth PO1 2DT, UK \\ 3 Portsmouth Hospitals NHS Trust, Queen Alexandra \\ Hospital, Cosham PO6 3LY, UK
}

submitted 28.6.2017

accepted after revision 20.10.2017

\author{
Bibliography \\ DOI https://doi.org/10.1055/a-0751-2613 | \\ Endoscopy International Open 2019; 07: E403-E411 \\ (c) Georg Thieme Verlag KG Stuttgart · New York \\ ISSN 2364-3722
}

Corresponding author

Professor Pradeep Bhandari, Portsmouth Hospitals NHS Trust, Queen Alexandra Hospital, Cosham PO6 3LY, UK Fax: +44-2392-286255

Pradeep.bhandari@porthosp.nhs.uk

\section{ABSTRACT}

Background Studies examining the impact of training modules on characterization of diminutive colonic polyps (DCP) show varying results.

Aim We aimed to assess the impact of a novel web-based training module on the accuracy of in vivo characterization of DCPs using different imaging modalities. Differences between groups with varying degrees of endoscopic experience were also assessed.

Methods In total, 90 images of 30 DCPs viewed with high definition white light (HDWL), i-Scan, and indigo carmine chromoendoscopy were included in an online test module. Testing was undertaken before and after completing a novel web-based in vivo characterization training module. In total, 21 subjects (medical students (MS), gastroenterology trainees (GT), and gastroenterology consultants (GC)) undertook the tests and training module.

Results No statistically significant difference in overall accuracy was found between the three groups either pre- (MS $59.1 \%$, GR 65.7\%, GC 62.4\%, $P=$ ns for all three comparisons) or post-training (MS 69.2\%, GR 71.1\%, GC $71.3 \%$, $P=$ ns for all three comparisons). Accuracy improved significantly for all three groups post-training $(P<0.001)$ as did interobserver agreement. No significant differences in accuracy between modalities were found pre-training (HDWL $64.8 \%$, i-Scan $60.0 \%$, chromoendoscopy $62.2 \%, P=n s$ ). Post-training accuracy with HDWL and chromoendoscopy was better than with i-Scan (HDWL $72.9 \%$ vs i-Scan $65.1 \%, \quad P=0.002$; i-Scan $65.1 \%$ vs chromoendoscopy $73.7 \%, P<0.001)$. The proportion of high confidence predictions increased from $25.7 \%$ to $41.5 \%$, with a high confidence prediction accuracy of $81.7 \%$.

Conclusions Skills for in vivo characterization of DCPs are not acquired through endoscopic experience alone. A novel web-based training intervention results in modest improvements in accuracy with further improvements likely to require more prolonged training.

\section{Introduction}

Small colonic polyp characterization has been identified as a key goal for novel endoscopic advanced imaging techniques and is an area of much research activity [1]. While expert endoscopists and those with an interest in in vivo diagnostic techniques can obtain high levels of accuracy, this level of performance has not generally been seen in studies involving non- expert endoscopists who have no particular experience or expertise in this area [2-6].

Hence, the issue of training in advanced diagnostic imaging techniques is of paramount importance if use of these techniques is to become part of everyday practice in the endoscopy community as a whole.

Several studies have examined the impact of brief training interventions on small colonic polyp (SCP) characterization. 
Significant improvements in the accuracy of diminutive colonic polyp (DCP) characterization have been demonstrated using advanced endoscopic imaging techniques (narrow-band imaging (NBI), i-Scan) following still image or video-based training interventions lasting 20-60 minutes [7-10]. In contrast, a similar study by Coe et al. showed no significant improvement following two 1-hour training sessions on the use of NBI for SCP characterization [11]. The impact of training on high definition white light (HDWL) polyp characterization and its relative performance compared to advanced imaging techniques and chromoendoscopy has not been widely studied. There is some evidence that the accuracy of HDWL characterization of DCPs can match that obtained using advanced imaging $[6,12,13]$.

\section{Aims}

This study aimed to examine the effect of a web-based training module on the accuracy of in vivo characterization of diminutive $(<5 \mathrm{~mm})$ colonic polyps using HDWL, i-Scan, and chromoendoscopy. Differences between groups with varying degrees of endoscopic experience were also assessed.

\section{Methods}

This work was formally assessed and deemed to be an evaluation of an educational tool and therefore did not require formal research ethics committee approval.

Images obtained during a previous study were used [12]. Informed consent was obtained from all patients for the use of anonymized images of polyps for future teaching and training purposes. High quality images of diminutive polyps viewed with HDWL, i-Scan 3 (Surface enhancement +3 , Contrast enhancement +2 , Tone enhancement-colon), and $0.2 \%$ indigo carmine chromoendoscopy were selected, with three images of each polyp used (one with each modality). In total, 30 polyps were included in this study, 15 adenomatous and 15 hyperplastic, which corresponds to the approximate proportions of each histological type found in DCPs in situ. Therefore, in total, 90 images of the 30 polyps were used in the study. Histopathological diagnosis as determined by a UK Bowel Cancer Screening Programme accredited histopathologist was used as the reference standard for all optical assessments.

Images were randomized using an online randomization tool (GraphPad) and incorporated into a web-based testing and training module hosted on the University of Portsmouth virtual learning platform (Moodle). Participants viewed each image in turn and were asked to predict polyp histology (adenomatous vs hyperplastic) and to also report their confidence level for each assessment based on the following confidence levels:

- $<70 \%$ certain = low confidence;

- $70-90 \%$ certain = medium confidence;

- $>90 \%$ certain $=$ high confidence.

All questions had to be answered to complete the training module. To avoid the influence of other participants, all tests and training were undertaken individually.
Following the initial 'pre-training' testing phase, subjects completed a novel web-based training tool on small colonic polyp characterization. The training module was developed using Prezi - a cloud-based presentation software program, and was designed to take 20 minutes to complete.

The training module covered several key areas:

- Outlining the two main types of DCPs (hyperplastic and adenomatous);

- Modes of assessing polyps in vivo - HDWL, i-Scan, and indigo carmine chromoendoscopy without magnification;

- Key features used for DCP characterization (vascularity, vascular patterns, and surface patterns) and which features were suggestive of each histological type:

- Hyperplastic polyps - paler, or similar in color to the surrounding mucosa; no visible surface patterns or large non-compact pits; no visible surface vessels, or a few thin thread-like surface vessels;

- Adenomatous polyps - more vascular than the surrounding mucosa; small compact regular pits, tubular or branched pits; dense regular vessels following the edges of pits;

- 20 image examples of all key features;

- An interactive "test" section where subjects were asked to predict histology, followed by feedback on key features visible and the histopathological diagnosis.

To avoid subject bias, the training module did not refer to which key features were visible with each imaging modality, nor whether any modality was likely to lead to a more accurate prediction of histology.

Screenshot images from the training module are shown in - Figs. 1 - 4 .

Following completion of the training module, subjects underwent a second test module using the same 90 polyp images presented in a different randomized order.

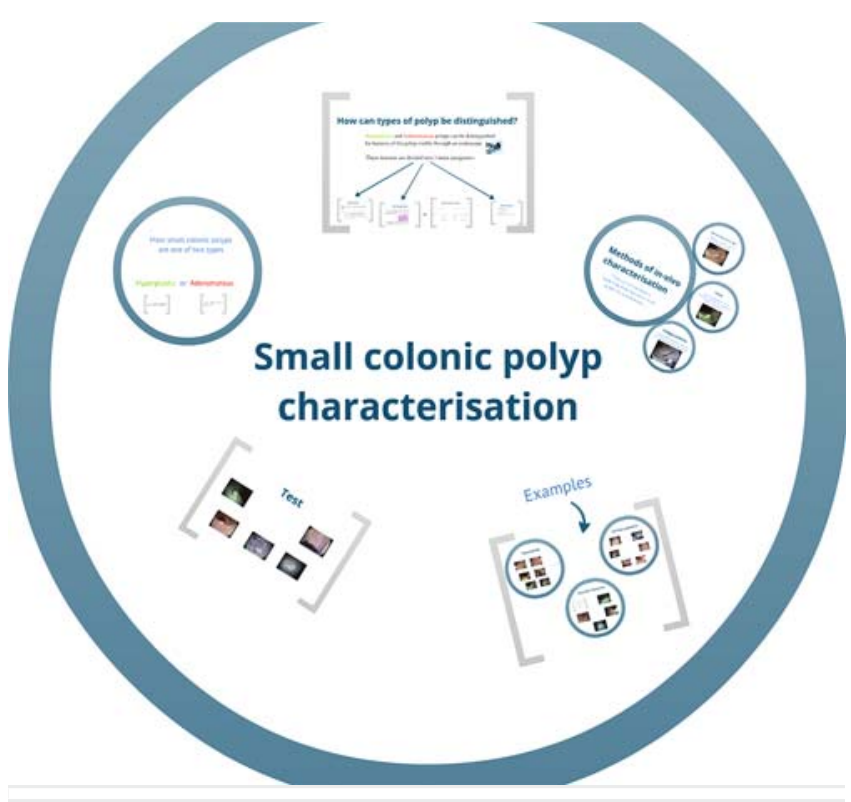

Fig. 1 Screenshot image from the web-based training module. 


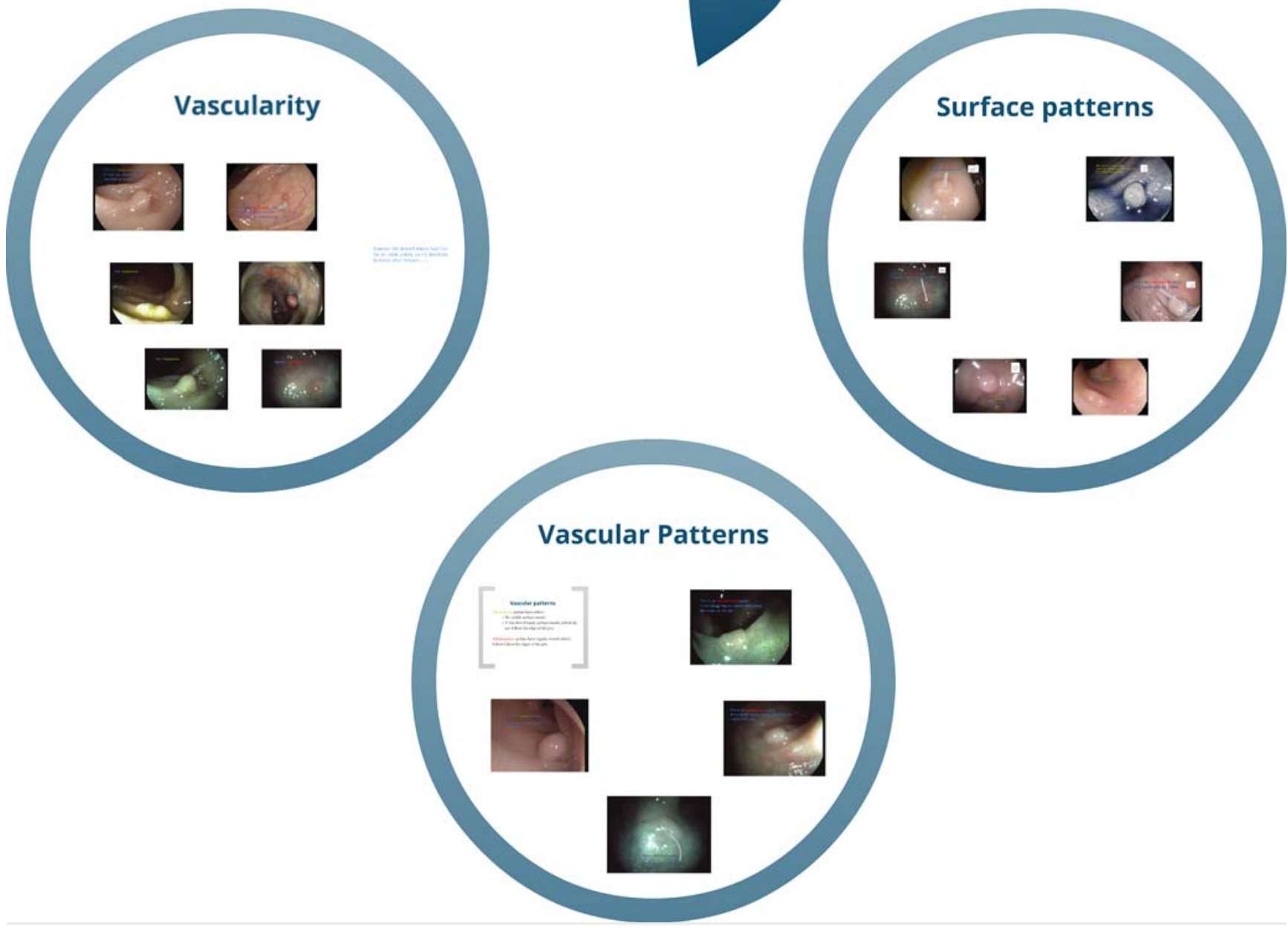

- Fig. 2 Screenshot image from the web-based training module.

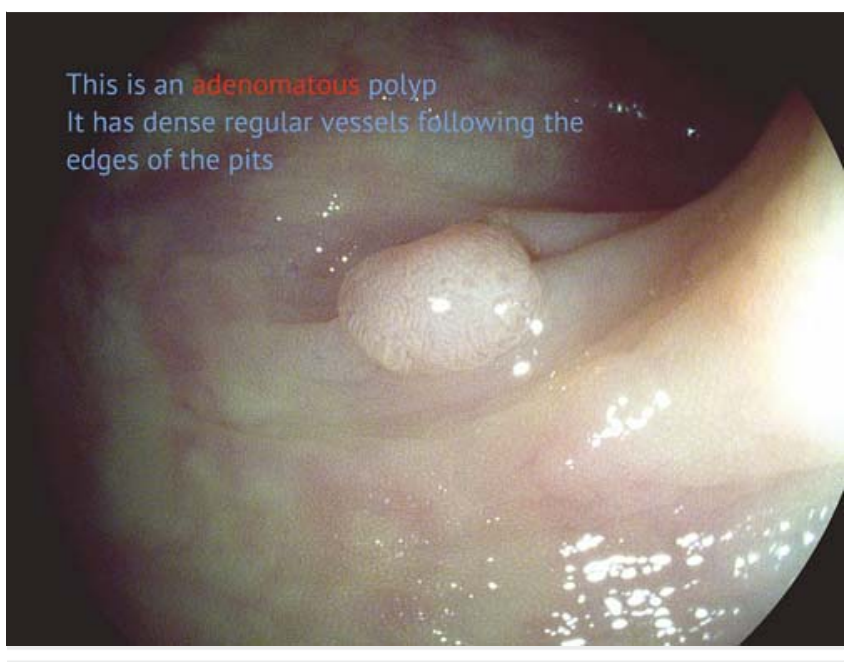

- Fig. 3 Screenshot image from the web-based training module.

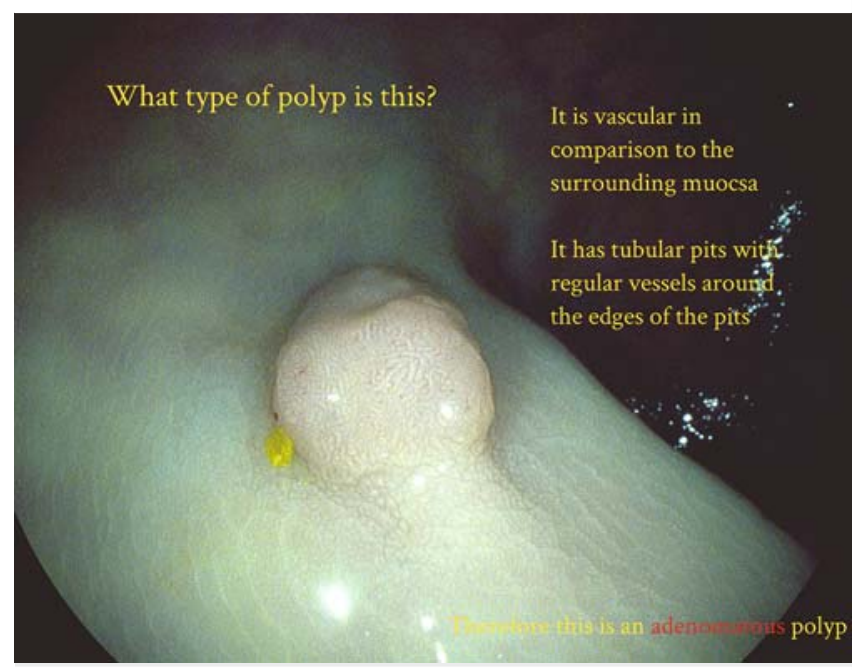

Fig. 4 Screenshot image from the web-based training module. 
Subjects were divided into three groups:

- 3 rd year medical students $(n=7)$

- First clinical year students. Limited experience of endoscopy with all having attended $0-2$ endoscopy lists in total. No previous training on DCP characterization.

- Gastroenterology Registrars ( $n=7)$

- Higher specialist gastroenterology trainees. All currently training in colonoscopy and polypectomy.

- No previous formal training in in vivo diagnostic techniques.

- Median lifetime colonoscopy cases performed: 120 (range 30-450).

- Gastroenterology Consultants ( $=7$ )

- No previous formal training in in vivo diagnostic techniques.

- Median lifetime colonoscopy cases performed: 3000 (range 1000-5000).

Two expert endoscopists with experience of over 5000 colonoscopies and extensive experience in in vivo diagnostic techniques, including extensive research experience in this field, also undertook the test module to validate the image library.

\section{Statistics}

The pre-training test was expected to demonstrate a difference in overall accuracy between the three subject groups. Based on pilot studies performed with similar subjects groups viewing polyp images, the predicted overall accuracy for each group was:

- Medical students $60 \%$;

- Registrars 70\%;

- Consultants $75 \%$.

Power calculations showed that seven subjects per group would be required to provide $80 \%$ power to demonstrate these differences with a significance level of $5 \%$.

Overall accuracy was expected to be similar in all three groups after training $[8,14,15]$. It was anticipated that, following training, there would be a small difference in accuracy between white light $(80 \%)$ and i-Scan/indigo carmine chromoendoscopy (85\%).
Comparisons between groups for mean accuracy, sensitivity, specificity, and negative predictive value (NPV) were performed by one-way ANOVA. In those comparisons where a significant difference was detected using ANOVA, post-hoc testing was performed to detect significant differences between pairs of groups using Hochberg's GT2 test (when sample sizes differed) or Tukey's test. Comparisons of mean accuracy by modality were also performed using one-way ANOVA and post-hoc Tukey's test. Differences in performance pre- and post-training were compared using McNemar's test. Differences in confidence levels between groups and modality were compared using the Pearson's chi-squared test. Odds ratios were calculated for two level confidence results (low/medium vs high) if a significant chi-squared statistic was detected.

Mean kappa values within groups pre- and post-training were also calculated and the accuracy of high confidence predictions was also assessed.

\section{Results}

\section{Overall accuracy}

Mean overall accuracy results in the pre-training test are shown in $>$ Table 1. No statistically significant differences were found between the three study groups pre-training ( $P>0.5$ for all three comparisons). The mean accuracy of the two experts was significantly higher than the three study groups $(P<0.01$ for all three comparisons).

In the post-training test, mean overall accuracy was significantly higher for all three groups compared to pre-training $(P<0.02$ for all three comparisons). Again, there were no statistically significant differences in accuracy between the three study groups post-training ( $P>0.5$ for all three comparisons) but mean accuracy remained significantly higher for the two experts ( $P<0.025$ for all three comparisons). Pre- and posttraining accuracy results for individual participants are shown in $>$ Fig. 5.

- Table 1 Pre-training and post-training accuracy by subject group.

\begin{tabular}{|l|l|l|c|}
\hline & $\begin{array}{l}\text { Accuracy pre-training: } \\
\text { Correct/Total, \% (95\%Cl) }\end{array}$ & $\begin{array}{l}\text { Accuracy post-training: } \\
\text { Correct/Total, \% (95\%Cl) }\end{array}$ & $\begin{array}{l}\text { P value pre-training vs } \\
\text { post-training }\end{array}$ \\
\hline Students & $372 / 630$, & $436 / 630$, & $<0.001$ \\
\hline Registrars & $59.1 \%(50.6-67.5 \%)$ & $69.2 \%(66.1-72.3 \%)$ & 0.013 \\
\hline Consultants & $414 / 630$, & $448 / 630$, & $<0.001$ \\
\hline Experts & $65.7 \%(60.8-70.7 \%)$ & $71.1 \%(67.4-74.9 \%)$ & \\
\hline & $393 / 630$, & $71.3 \%(64.7-77.9 \%)$ & \\
\hline
\end{tabular}




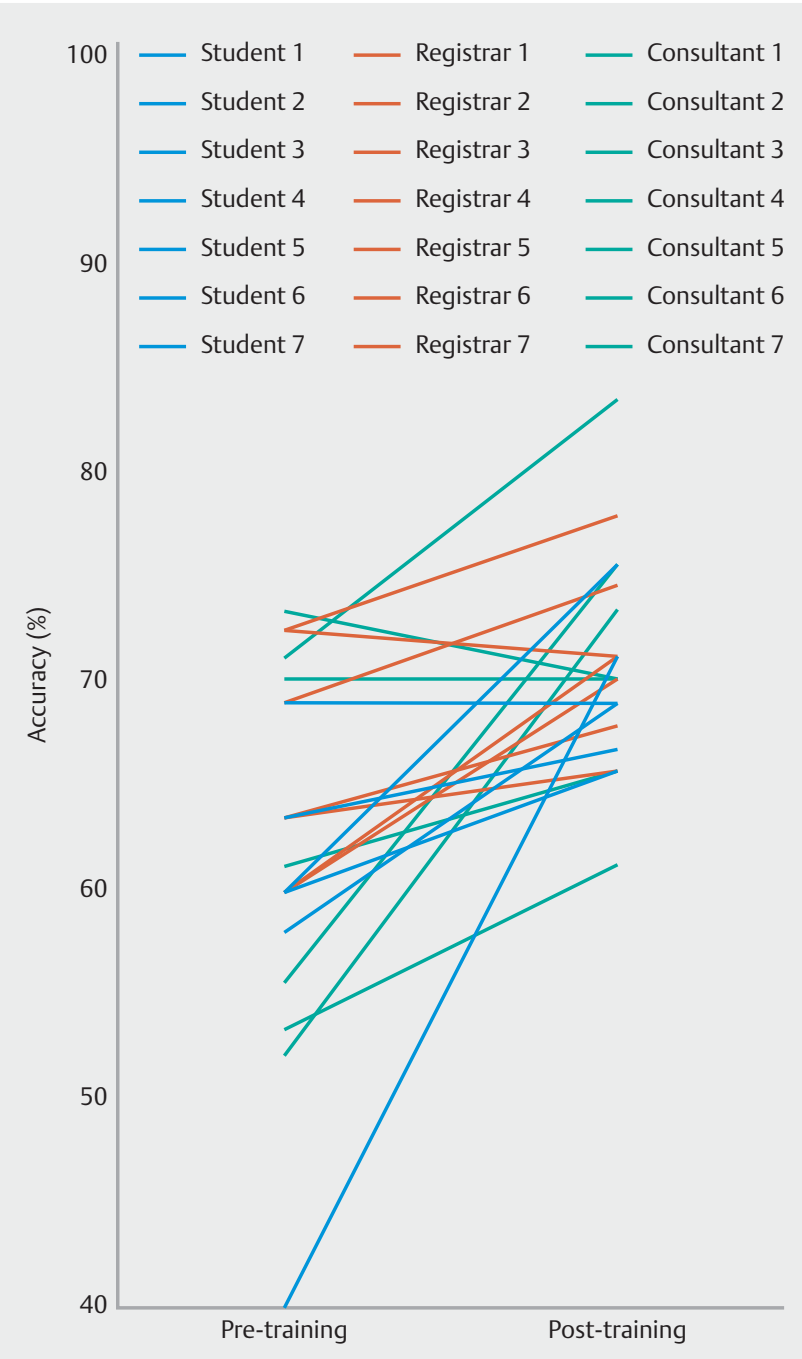

Fig. 5 Individual participant accuracy scores pre- and post-training.

\section{Sensitivity, specificity, and negative predictive value}

In analysis of sensitivity, specificity, and negative predictive value (NPV) for adenomatous histology, no statistically significant differences were found between the three groups either pre- or post-training ( $\triangleright$ Tables $2-4)$.

\section{Interobserver agreement}

Mean kappa values for each group pre- and post-training were calculated ( $\bullet$ Table 5 ). Agreement pre-training in the student group was slight, and fair in the registrar and consultant groups. There was a significant improvement in mean kappa for all three groups post-training. Post-training mean kappa was fair for the consultant group and moderate for the student and registrar groups.

\section{Performance by modality}

Accuracy rates achieved when assessing images with each of the three modalities (HDWL/i-Scan/indigo carmine chromoendoscopy) were compared pre- and post-training ( $\bullet$ Table 6 and - Table 7). No significant difference in mean accuracy between the three modalities was found pre-training. Post-training mean accuracy for HDWL and chromoendoscopy images was significantly higher than for i-Scan images.

Following training, accuracy for HDWL and chromoendoscopy images improved significantly $(P=0.002$ and $P<0.001$, respectively) compared to pre-training. However, accuracy with i-Scan images did not improve significantly post-training $(P=0.074)$.

\section{Confidence ratings by subject group}

Pre-training, there were significant differences between the three groups in the spread of confidence ratings. Students rated few of their predictions as high confidence (only 3.7\% overall). Registrars and consultants made more high confidence predictions, although the majority of their predictions were still rated as low or medium confidence ( $\bullet$ Table 8 and - Table 9).

Post-training, there remained a significant difference between the three groups in confidence levels ( $\bullet$ Table 9 ). However, the proportion of predictions made with high confidence by students had risen from $3.7 \%$ to $30.5 \%$, and with medium confidence from $28.4 \%$ to $39.2 \%$. The proportion of high confidence predictions also increased significantly for the registrar and consultant groups but to a lesser degree ( $\triangleright$ Table 9 and $\triangleright$ Table 10). Registrars and consultants remained more likely to make a high confidence prediction than students but the difference was much less marked than seen pre-training $(\vee$ Table 8 and $\triangleright$ Table 9 ).

- Table 2 Pre-training and post-training sensitivity by group with comparisons between groups.

\begin{tabular}{|c|c|c|c|c|c|}
\hline & $\begin{array}{l}\text { Sensitivity pre-training: } \\
\text { Correct/Total, \% ( } 95 \% \mathrm{Cl} \text { ) }\end{array}$ & $\begin{array}{l}P \text { value for } \\
\text { comparison be- } \\
\text { tween groups }\end{array}$ & $\begin{array}{l}\text { Sensitivity post-training: } \\
\text { Correct/Total, } \%(95 \% \mathrm{Cl})\end{array}$ & $\begin{array}{l}P \text { value for } \\
\text { comparison be- } \\
\text { tween groups }\end{array}$ & $\begin{array}{l}P \text { value pre-training } \\
\text { vs post-training }\end{array}$ \\
\hline Students & $\begin{array}{l}175 / 315 \\
55.6 \%(36.8-74.3 \%)\end{array}$ & \multirow[t]{3}{*}{0.285} & $\begin{array}{l}223 / 315 \\
70.8 \%(63.1-78.5 \%)\end{array}$ & \multirow[t]{3}{*}{0.520} & $<0.001$ \\
\hline Registrars & $\begin{array}{l}218 / 315 \\
69.2 \%(59.8-78.7 \%)\end{array}$ & & $\begin{array}{l}222 / 315 \\
70.5 \%(65.6-75.3 \%)\end{array}$ & & 0.789 \\
\hline Consultants & $\begin{array}{l}203 / 315 \\
64.4 \%(50.3-78.6 \%)\end{array}$ & & $\begin{array}{l}234 / 315 \\
74.3 \%(68.4-80.2 \%)\end{array}$ & & 0.007 \\
\hline
\end{tabular}


- Table 3 Pre-training and post-training specificity by group with comparisons between groups.

\begin{tabular}{|c|c|c|c|c|c|}
\hline & $\begin{array}{l}\text { Specificity pre-training: } \\
\text { Correct/Total, \% ( } 95 \% \mathrm{Cl})\end{array}$ & $\begin{array}{l}P \text { value for } \\
\text { comparison be- } \\
\text { tween groups }\end{array}$ & $\begin{array}{l}\text { Specificity post-training: } \\
\text { Correct/Total, \% ( } 95 \% \mathrm{Cl})\end{array}$ & $\begin{array}{l}P \text { value for } \\
\text { comparison be- } \\
\text { tween groups }\end{array}$ & $\begin{array}{l}P \text { value pre-training } \\
\text { vs post-training }\end{array}$ \\
\hline Students & $\begin{array}{l}197 / 315 \\
62.5 \%(49.6-75.5 \%)\end{array}$ & \multirow[t]{3}{*}{0.973} & $\begin{array}{l}213 / 315 \\
67.6 \%(55.9-79.4 \%)\end{array}$ & \multirow[t]{3}{*}{0.825} & 0.211 \\
\hline Registrars & $\begin{array}{l}195 / 315 \\
61.9 \%(49.8-74.0 \%)\end{array}$ & & $\begin{array}{l}226 / 315 \\
71.7 \%(64.7-78.8 \%)\end{array}$ & & 0.010 \\
\hline Consultants & $\begin{array}{l}190 / 315 \\
60.3 \%(37.0-83.6 \%)\end{array}$ & & $\begin{array}{l}214 / 315 \\
67.9 \%(50.6-85.2 \%)\end{array}$ & & 0.045 \\
\hline
\end{tabular}

- Table 4 Pre-training and post-training negative predictive value (NPV) by group with comparisons between groups.

\begin{tabular}{|c|c|c|c|c|c|}
\hline & $\begin{array}{l}\text { NPV pre-training: } \\
\text { Mean \% (95\%Cl) }\end{array}$ & $\begin{array}{l}P \text { value for } \\
\text { comparison be- } \\
\text { tween groups }\end{array}$ & $\begin{array}{l}\text { NPV post-training: } \\
\text { Mean \% }(95 \% \mathrm{Cl})\end{array}$ & $\begin{array}{l}P \text { value for } \\
\text { comparison be- } \\
\text { tween groups }\end{array}$ & $\begin{array}{l}P \text { value pre-training } \\
\text { vs post-training }\end{array}$ \\
\hline Students & $59.8 \%(51.7-67.9 \%)$ & \multirow[t]{3}{*}{0.220} & $70.2 \%(67.4-73.1 \%)$ & \multirow[t]{3}{*}{0.523} & 0.003 \\
\hline Registrars & $67.2 \%(61.4-73.0 \%)$ & & $70.9 \%(67.4-74.4 \%)$ & & 0.294 \\
\hline Consultants & $62.7 \%(55.4-70.0 \%)$ & & $72.3 \%(69.2-75.4 \%)$ & & 0.014 \\
\hline
\end{tabular}

- Table 5 Interobserver agreement by group pre- and post-training.

\begin{tabular}{|c|c|c|c|}
\hline & Mean pre-training kappa $(95 \% \mathrm{Cl})$ & Mean post-training kappa $(95 \% \mathrm{Cl})$ & Pre vs post kappa change, $P$ value \\
\hline Students & $0.106(-0.009-0.222)$ & $0.472(0.417-0.528)$ & $<0.001$ \\
\hline Registrars & $0.298(0.239-0.258)$ & $0.541(0.502-0.581)$ & $<0.001$ \\
\hline Consultants & $0.216(0.128-0.304)$ & $0.371(0.321-0.422)$ & 0.004 \\
\hline
\end{tabular}

- Table 6 Mean accuracy pre-training by modality.

\begin{tabular}{|c|c|c|}
\hline & $\begin{array}{l}\text { Accuracy pre-training: } \\
\text { Correct/Total, \% ( } 95 \% \mathrm{Cl})\end{array}$ & $P$ value \\
\hline HDWL & $408 / 630,64.8 \%(59.1-70.4 \%)$ & \multirow[t]{3}{*}{0.317} \\
\hline i-Scan & $378 / 630,60.0 \%(56.1-63.9 \%)$ & \\
\hline $\begin{array}{l}\text { Chromo- } \\
\text { endoscopy }\end{array}$ & $392 / 630,62.2 \%(58.2-66.2 \%)$ & \\
\hline
\end{tabular}

\section{Accuracy of high confidence predictions}

When only high confidence predictions in the post-training test were analyzed, mean accuracy was significantly higher for all three study groups ( $P<0.01$ for all three groups) ( $\triangleright$ Table 11). There was no difference in the mean accuracy of high confidence predictions between the three study groups. Accuracy of high confidence predictions by the two experts was higher that their overall performance $(93.6 \%$ vs $86.7 \%$ ) but this did not quite reach statistical significance $(P=0.052)$.
- Table 7 Mean accuracy post-training by modality.

\begin{tabular}{|l|l|l|l|}
\hline & $\begin{array}{l}\text { Accuracy post- } \\
\text { training: } \\
\text { Correct/Total, } \% \\
(\mathbf{9 5 \% C l )}\end{array}$ & $\begin{array}{l}\text { Comparison } \\
\text { vs HDWL } \boldsymbol{P} \\
\text { value }\end{array}$ & $\begin{array}{l}\text { Comparison } \\
\text { vs i-Scan } \boldsymbol{P} \\
\text { value }\end{array}$ \\
\hline HDWL & $\begin{array}{l}459 / 630,72.9 \% \\
(70.2-75.5 \%)\end{array}$ & & \\
\hline i-Scan & $\begin{array}{l}410 / 630,65.1 \% \\
(61.3-68.8 \%)\end{array}$ & 0.002 & \\
\hline Chromoen- & $\begin{array}{l}464 / 630,73.7 \% \\
(70.7-76.6 \%)\end{array}$ & 0.927 & $<0.001$ \\
\hline doscopy & & \\
\hline HDWL, high definition white light. & & \\
\hline
\end{tabular}

\section{Discussion}

This large study examined the baseline in vivo characterization skills for assessing DCPs amongst three groups with widely varying experience of endoscopy and polypectomy, plus the impact of a novel web-based training module. Differences be- 
- Table 8 Prediction confidence ratings pre-training.

\begin{tabular}{|l|l|l|l|l|}
\hline & \multicolumn{2}{|l|}{ Confidence in predictions pre-training } & P value \\
\hline & Low & Medium & High & $<0.001$ \\
\hline Students & $428(67.9 \%)$ & $179(28.4 \%)$ & $23(3.7 \%)$ & $195(31.0 \%)$ \\
\hline Registrars & $214(34.0 \%)$ & $221(35.1 \%)$ & $267(42.4 \%)$ \\
\hline Consultants & $187(29.7 \%)$ & $176(27.9 \%)$ & $485(25.7 \%)$ \\
\hline All subjects & $829(43.9 \%)$ & $576(30.5 \%)$ & $125(69.4 \%)$ & \\
\hline Experts & $19(10.6 \%)$ & $36(20.0 \%)$ & \\
\hline
\end{tabular}

- Table 9 Prediction confidence ratings post-training.

\begin{tabular}{|l|l|l|l|l|}
\hline & \multicolumn{2}{|l|}{ Confidence in predictions post-training } & P value \\
\hline & Low & Medium & High & $<0.001$ \\
\hline Students & $191(30.3 \%)$ & $247(39.2 \%)$ & $192(30.5 \%)$ & $284(45.1 \%)$ \\
\hline Registrars & $139(22.1 \%)$ & $207(32.9 \%)$ & $309(49.0 \%)$ \\
\hline Consultants & $145(23.0 \%)$ & $176(27.9 \%)$ & $785(41.5 \%)$ & \\
\hline All subjects & $475(25.1 \%)$ & $630(33.3 \%)$ & \\
\hline
\end{tabular}

- Table 10 Odds ratio of high confidence prediction pre- vs post-training.

\begin{tabular}{|c|c|c|c|}
\hline & Low/Medium & High & $\begin{array}{l}\text { Odds Ratio high confidence prediction } \\
\text { ( } 95 \% \text { confidence interval) }\end{array}$ \\
\hline \multicolumn{4}{|c|}{ Prediction confidence-Students } \\
\hline - Pre-training & 607 & 23 & 1.0 \\
\hline - Post-training & 438 & 192 & $11.57(7.38-18.14)$ \\
\hline \multicolumn{4}{|c|}{ Prediction confidence-Registrars } \\
\hline - Pre-training & 435 & 195 & 1.0 \\
\hline - Post-training & 346 & 284 & $1.83(1.45-2.31)$ \\
\hline \multicolumn{4}{|c|}{ Prediction confidence-Consultants } \\
\hline - Pre-training & 363 & 267 & 1.0 \\
\hline - Post-training & 321 & 309 & $1.31(1.05-1.63)$ \\
\hline
\end{tabular}

- Table 11 Mean accuracy of high confidence predictions.

\begin{tabular}{|c|c|c|}
\hline & $\begin{array}{l}\text { High confidence accuracy } \\
\text { post-training } \%(95 \% \mathrm{Cl})\end{array}$ & $P$ value \\
\hline Students & $82.4 \%(74.3-90.5 \%)$ & \multirow[t]{3}{*}{0.785} \\
\hline Registrars & $79.8 \%(76.2-83.5 \%)$ & \\
\hline Consultants & $82.9 \%(72.2-93.5 \%)$ & \\
\hline Experts & $93.6 \%(53.6-100 \%)$ & \\
\hline
\end{tabular}

tween three endoscopic modalities, HDWL, i-Scan, and indigo carmine chromoendoscopy were also examined.

Perhaps the most striking results are the pre-training accuracy rates, which showed no statistically significant difference in accuracy between the three participant groups. Accuracy amongst medical students, who had observed at most a handful of colonoscopies, did not differ from that of experienced endoscopists. One would logically assume that the experience of performing several thousand colonoscopies, and hundreds/ thousands of polypectomies during those procedures, would lead to the acquisition of in vivo diagnostic skills amongst consultants, but these results indicate that is not necessarily the case. Studies assessing the accuracy of colonic lesion assess- 
ment, including early colorectal cancers, have similarly found that experienced endoscopists performed no better than trainees or non-endoscopist nurses $[16,17]$.

Following the training module, performance improved significantly for all groups, as did agreement between subjects and confidence in predictions. However, accuracy remained significantly below that of expert endoscopists. Although not specifically addressed by this study, the ASGE PIVI criteria for optical diagnosis of DCPs are unlikely to have been met by any of the groups following training. To reach the levels of accuracy shown by experts is likely to require an ongoing period of practicing optical diagnostic skills in vivo with regular review and feedback of performance.

Previous studies have suggested that accurate characterization of DCPs using advanced endoscopic imaging can be learnt following picture-based training lasting 15-20 minutes [7, 8]. Similar video-based studies reported by Neumann et al. and Patel et al., including feedback during training, elicited improvements in diagnostic accuracy $[9,10]$. This suggests that feedback on performance is a key component of learning optical diagnosis skills. Both of these studies gave feedback after each question in their test modules and hence ongoing learning occurred through the testing phase. Feedback was not given in the current study, and if included, may have improved performance.

In contrast, a similar study by Coe et al. examined the impact of two 1-hour training sessions on the use of $\mathrm{NBI}$ for small colon polyp characterization. They assessed the real-time in vivo characterization accuracy of 15 endoscopists who were split into those receiving training, and a control group, who did not receive training [11]. No significant improvement in prediction of polyp histology, or surveillance intervals, occurred following the training sessions.

Studies of brief training interventions show varying results in the accuracy achieved by participants post-training. In this current study, post-training accuracy was around $70 \%$ for all three groups, which may suggest that training endoscopists to achieve high accuracy rates is more difficult than suggested in other studies. In another study using i-Scan, participants achieved $94 \%$ accuracy for the final set of study images [9]. Most other training studies have used NBI and reported that post-training accuracy levels vary from $80 \%[11,18]$ to around $90 \%[7,8,10]$. Several factors could influence post-training accuracy: ability of participants to acquire new skills, effectiveness of the training module, imaging modality used, and difficulty of the test module.

As demonstrated in $\mathbf{F i g . 5}$, there was notable variability between participants in the improvement in accuracy following training. Of the 21 participants, 17 improved their scores following training, three remained the same and one actually scored lower post-training. This variability may be explained by differences between subjects in ability and motivation to acquire new skills.

The training module devised for this study covered several factors used for optical diagnosis-vascularity, surface patterns, and vascular patterns. NBI training modules are likely to just cover vascularity and vascular patterns and hence may be simpler and less likely to confuse participants unfamiliar with advanced imaging techniques. The training module in this study also covered three modalities - HDWL, i-Scan, and indigo carmine chromoendoscopy, which may have added complexity and the potential to overload participants.

The test module used in this study may have been more challenging than those used in other studies. In a similar study performed by Ignjatovic et al., an expert group (one of whom also participated in this study) achieved $95 \%$ accuracy [7], compared to $86.7 \%$ in this study. We selected high quality images for this study but did not restrict selection to polyps which showed very obvious and typical features of either hyperplastic or adenomatous DCPs, and hence some of the polyps included may have proven to be more "difficult" than others. There may be a propensity in studies of this type for researchers to select images which demonstrate very apparent features of adenomatous and hyperplastic polyps, which may not represent the full spectrum of DCPs found in clinical practice. This study used still images for the test module whereas other studies have used short videos $[18,19]$ which may enable a more accurate assessment to be made.

Whereas most other studies have only assessed training in just one endoscopic modality, this study compared three different endoscopic modalities, and participants' ability to acquire optical diagnosis assessment skills with each of them. Most published studies suggest that advanced imaging techniques or chromoendoscopy are superior to white-light endoscopy in the assessment of SCPs/DCPs [20-22]. The results of this study suggest that this may not be the case for non-expert endoscopists and novices (medical students). Following the training module, accuracy with HDWL was not significantly different to that with chromoendoscopy and was actually significantly higher than accuracy with i-Scan. This may suggest that optical diagnosis skills with HDWL are easier to acquire than with i-Scan. Endoscopists may have also been unfamiliar with the i-Scan image, and despite undergoing the training module, found it more difficult to interpret than a white light image that they would be used to seeing in everyday practice.

Further studies are required comparing advanced imaging techniques with HDWL imaging to explore whether HDWL is a viable alternative to NBI/Fujinon intelligent chromoendoscopy (FICE)/i-Scan for optical diagnosis. Ongoing research is also required to determine what further training is needed for a nonexpert endoscopist to achieved optical diagnostic accuracy that can meet the ASGE PIVI criteria. This will undoubtedly require a period of monitored in vivo training and feedback over a period of time, in addition to computer/web-based initial training. National and international endoscopy societies will need to set out a clear training and certification process for optical diagnosis for this to become widespread practice. At present, optical diagnosis skills do not form part of the curriculum for higher gastroenterology trainees in the UK (Joint Royal Colleges of Physicians Training Board. Specialty Training Curriculum for Gastroenterology August 2010 (Amendments August 2013). https:// www.jrcptb.org.uk/sites/default/files/2010\%20Gastroenterology\%20\%28amendment\%202013\%29_0.pdf). Clearly, it would make sense to teach these skills alongside technical skills in 
endoscopy so that newly qualified consultants have acquired them before entering independent practice.

\section{Competing interests}

None

\section{References}

[1] Rex DK, Kahi C, O’Brien M et al. The American Society for Gastrointestinal Endoscopy PIVI (Preservation and Incorporation of Valuable Endoscopic Innovations) on real-time endoscopic assessment of the histology of diminutive colorectal polyps. Gastrointest Endosc 2011; 73: $419-422$

[2] Paggi S, Rondonotti E, Amato A et al. Resect and discard strategy in clinical practice: a prospective cohort study. Endoscopy 2012; 44: $899-904$

[3] Kuiper T, Marsman WA, Jansen JM et al. Accuracy for optical diagnosis of small colorectal polyps in nonacademic settings. Clin Gastroenterol Hepatol 2012; 10: 1016 - 1020; quiz e79

[4] Ladabaum U, Fioritto A, Mitani A et al. Real-time optical biopsy of colon polyps with narrow band imaging in community practice does not yet meet key thresholds for clinical decisions. Gastroenterology 2013; 144: $81-91$

[5] Schachschal G, Mayr M, Treszl A et al. Endoscopic versus histological characterisation of polyps during screening colonoscopy. Gut 2014; 63: $458-465$

[6] Vu HT, Sayuk GS, Hollander TG et al. Resect and discard approach to colon polyps: real-world applicability among academic and community gastroenterologists. Dig Dis Sci 2015; 60: 502-508

[7] Ignjatovic A, Thomas-Gibson S, East JE et al. Development and validation of a training module on the use of narrow-band imaging in differentiation of small adenomas from hyperplastic colorectal polyps. Gastrointest Endosc 2011; 73: 128-133

[8] Raghavendra M, Hewett DG, Rex DK. Differentiating adenomas from hyperplastic colorectal polyps: narrow-band imaging can be learned in 20 minutes. Gastrointest Endosc 2010; 72: 572-576

[9] Neumann H, Vieth M, Fry LC et al. Learning curve of virtual chromoendoscopy for the prediction of hyperplastic and adenomatous colorectal lesions: a prospective 2-center study. Gastrointest Endosc 2013; 78: $115-120$

[10] Patel SG, Rastogi A, Austin G et al. Gastroenterology trainees can easily learn histologic characterization of diminutive colorectal polyps with narrow band imaging. Clin Gastroenterol Hepatol 2013; 11: $997-1003$ e 1
[11] Coe SG, Thomas C, Crook J et al. Colorectal surveillance interval assignment based on in vivo prediction of polyp histology: impact of endoscopic quality improvement program. Gastrointest Endosc 2012; 76: $118-125$ e1

[12] Basford PJ, Longcroft-Wheaton G, Higgins B et al. High-definition endoscopy with i-Scan for evaluation of small colon polyps: the HiSCOPE study. Gastrointest Endosc 2014; 79: 111 - 118

[13] Apel D, Jakobs R, Schilling D et al. Accuracy of high-resolution chromoendoscopy in prediction of histologic findings in diminutive lesions of the rectosigmoid. Gastrointest Endosc 2006; 63: 824-828

[14] Higashi R, Uraoka T, Kato J et al. Diagnostic accuracy of narrow-band imaging and pit pattern analysis significantly improved for less-experienced endoscopists after an expanded training program. Gastrointest Endosc 2010; 72: 127 - 135

[15] Rastogi A, Pondugula K, Bansal A et al. Recognition of surface mucosal and vascular patterns of colon polyps by using narrow-band imaging: interobserver and intraobserver agreement and prediction of polyp histology. Gastrointest Endosc 2009; 69: 716 - 722

[16] Basford PJ, Longcroft-Wheaton G, Higashi R et al. Colonic lesion characterisation skills among UK endoscopists and the impact of a brief training intervention. Frontline Gastroenterol 2017; 8: 2 -7

[17] van der Vlugt M, van Doorn SC, Wang J et al. Optical diagnosis of malignant colorectal polyps: is it feasible? Endosc Int Open 2016; 4: E778-783

[18] Rastogi A, Rao DS, Gupta N et al. Impact of a computer-based teaching module on characterization of diminutive colon polyps by using narrow-band imaging by non-experts in academic and community practice: a video-based study. Gastrointest Endosc 2014; 79 : $390-398$

[19] Sinh P, Gupta N, Rao DS et al. Community gastroenterologists can learn diminutive colon polyp histology characterization with narrow band imaging by a computer-based teaching module. Dig Endosc 2015; $27: 374-380$

[20] Wanders LK, East JE, Uitentuis SE et al. Diagnostic performance of narrowed spectrum endoscopy, autofluorescence imaging, and confocal laser endomicroscopy for optical diagnosis of colonic polyps: a meta-analysis. Lancet Oncol 2013; 14: 1337-1347

[21] Fu KI, Sano Y, Kato S et al. Chromoendoscopy using indigo carmine dye spraying with magnifying observation is the most reliable method for differential diagnosis between non-neoplastic and neoplastic colorectal lesions: a prospective study. Endoscopy 2004; 36: $1089-1093$

[22] Chiu HM, Chang CY, Chen CC et al. A prospective comparative study of narrow-band imaging, chromoendoscopy, and conventional colonoscopy in the diagnosis of colorectal neoplasia. Gut 2007; 56 : $373-379$ 